\title{
Article \\ Simulation and Evaluation of the Radiation Dose Deposited in Human Tissues by Atmospheric Neutrons
}

\author{
Ernesto Ortiz ${ }^{1,2, *(\mathbb{D})}$, Blanca Mendoza ${ }^{1,3}$, Carlos Gay ${ }^{2}$, Victor Manuel Mendoza ${ }^{2}$, Marni Pazos ${ }^{2}$ \\ and Rene Garduño ${ }^{2}$ \\ 1 Escuela Nacional de Ciencias de la Tierra, Universidad Nacional Autónoma de México, Coyoacán, \\ Ciudad de México 04510, Mexico; blanca@igeofisica.unam.mx \\ 2 Centro de Ciencias de la Atmósfera, Universidad Nacional Autónoma de México, Coyoacán, \\ Ciudad de México 04510, Mexico; cgay@unam.mx (C.G.); victor@atmosfera.unam.mx (V.M.M.); \\ marni@unam.mx (M.P.); rene@atmosfera.unam.mx (R.G.) \\ 3 Instituto de Geofísica, Universidad Nacional Autónoma de México, Coyoacán, \\ Ciudad de México 04510, Mexico \\ * Correspondence: eortiz@encit.unam.mx
}

check for updates

Citation: Ortiz, E.; Mendoza, B.; Gay, C.; Mendoza, V.M.; Pazos, M.; Garduño, R. Simulation and Evaluation of the Radiation Dose Deposited in Human Tissues by Atmospheric Neutrons. Appl. Sci. 2021, 11, 8338. https://doi.org/ 10.3390/app11188338

Academic Editors: Roberta Sparvoli and Francesco Palma

Received: 22 June 2021

Accepted: 31 August 2021

Published: 9 September 2021

Publisher's Note: MDPI stays neutral with regard to jurisdictional claims in published maps and institutional affiliations.

Copyright: (c) 2021 by the authors. Licensee MDPI, Basel, Switzerland. This article is an open access article distributed under the terms and conditions of the Creative Commons Attribution (CC BY) license (https:// creativecommons.org/licenses/by/ $4.0 /)$.

\begin{abstract}
The evaluation of the radiation dose (RD) deposited by atmospheric neutrons in human tissues is of vital importance due to the potential damages that over exposure to this radiation may cause to human health. The goal of this work was to obtain the RD that atmospheric neutrons with energy from 1 to $1000 \mathrm{MeV}$ deposit in tissues of the human body (blood, adipose, bone and brain) as a function of both altitude and latitude. With the help of the Geant 4 software, we developed a numerical simulation that allowed us to reach our goal; atmospheric neutron fluxes were obtained from the Excel-Based Program for Calculating Atmospheric Cosmic-Ray Spectrum (EXPACS). We found that the RD deposited by atmospheric neutrons increases with the increase in altitude and latitude, e.g., for an altitude of high mountain $(4 \mathrm{~km})$, the RD is increased $\sim 19$ times; while, for an altitude of commercial flights (10 km), the RD is increased 156 times; in both cases, regarding the $\mathrm{RD}$ at sea level. We also found that, in the range of energies from 1 to $100 \mathrm{MeV}$, the RD deposited in the bone tissue sample is considerably lower that the RD deposited in the blood, adipose and brain tissue samples. On the other hand, for the range of energies between 200 and $1000 \mathrm{MeV}$, the RD deposited in the bone tissue sample is considerably greater that the RD deposited in the blood, adipose and brain tissue samples.
\end{abstract}

Keywords: atmospheric neutrons; radiation dose; passengers and flight crew; cosmic rays

\section{Introduction}

The atmospheric air showers are initiated by high-energy primary cosmic rays that enter the atmosphere isotropically from outer space, producing a large number of secondaries particles in a serie of successive collisions with target nuclei of the atmospheric constituents [1]. The primary cosmic rays which consists predominantly of protons, alpha particles and heavier nuclei are influenced by the galactic, the interplanetary, the magnetospheric and the geomagnetic magnetic fields while approaching the Earth [2].

In addition, the incoming cosmic rays with energies below about $20 \mathrm{GeV}$ are modulated by the interplanetary magnetic field which is embedded in the solar wind [3], the expanding magnetized plasma generated by the Sun, which decelerates and partially excludes the lower energy cosmic rays from the inner solar system. There is a significant anticorrelation between solar activity and the intensity of the cosmic rays with the energies already mentioned.

Atmospheric neutrons are secondary particles resulting from interactions of primaries with nuclei of air constituents. It is useful to distinguish low-energy neutrons with energies less than about $10 \mathrm{MeV}$ from high-energy neutrons. Low-energy neutrons are mostly 
evaporation products of excited nuclei and manifest an isotropic angular distribution. High-energy neutrons can be produced by hadronic collisions but also in charge exchange reactions of leading particles at very high energies, the angular distribution of high energy neutrons is anisotropic [2]. In addition, when high-energy particles collide with the atoms in the aircraft material, producing a local shower of particles, including electrons, lighter ions, gamma rays, and neutrons that are dangerous to human health [4].

Because the neutrons are produced by charged particles they inherit and also exhibit both latitude and longitude effects. Furthermore the flux of the low-energy neutron component is modulated by the solar cycle; in addition, strong solar flares may cause significant neutron fluxes in the atmosphere [2], sporadic enhancements of solar cosmic ray fluxes are also caused by coronal mass ejection, in these events the particles are accelerated to energies of hundreds of $\mathrm{MeV}$ up to several $\mathrm{GeV}[5,6]$.

The secondary energetic particles interacting with the human body may potentially cause an increase in cancer risk as the dose equivalent exposure increases, particularly in passengers and flight crew [7]. High RD may cause the breaks, potentially lethal to the cell by damaging the DNA strands, while low RD of ionizing radiations seems to have carcinogenic effects, even after years or decades, both in the exposed individuals and in subsequent generations [8]. The atmospheric neutron component of this complex radiation field, in particular, holds special interest in the cancer research community. At aircraft altitude (flight level around $10 \mathrm{~km}$ ) of the total radiation dose deposited by secondary particles, the contribution of neutrons is around 40\% [9].

The potential negative effect of atmospheric neutrons on human health is the motivation to carry out the present work, in this, we developed a numerical simulation using Geant4 software, with which, we calculated the radiation dose (RD) deposited by atmospheric neutrons in various tissues of the human body as function of the fluxes of these particles for various altitudes and latitudes; however, in all these geographical points we have maintained the same longitude $\left(0^{\circ}\right)$, since these will allow us to show the great variations of the $\mathrm{RD}$ as a function of altitude and latitude.

In this work, we have focused on the RD that atmospheric neutrons deposit in human tissues, if the readers want to know the effects of charged particles, they can read for example $[6,8,10]$.

\section{Materials and Methods: Setup of the Simulation}

Excel-Based Program for Calculating Atmospheric Cosmic-Ray Spectrum (EXPACS) calculate cosmic ray fluxes of neutrons, protons, muons, electrons, positrons, and photons nearly anytime and anywhere in the Earth's atmosphere [11].

In Figure 1, we show the atmospheric neutron spectra for various altitudes obtained with EXPACS for geographical points with a latitude of $0^{\circ}$ and a longitude of $0^{\circ}$. These data were calculated for the year 2020, period of minimum solar activity.

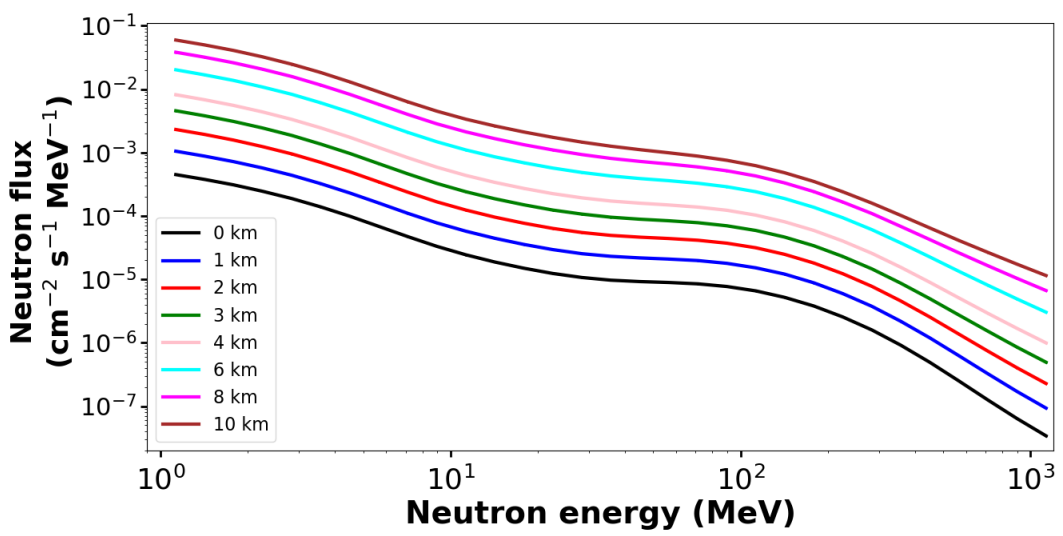

Figure 1. Atmospheric neutron spectra for various altitudes at geographical points with a latitude of $0^{\circ}$ and a longitude of $0^{\circ}$. See text for more details. 
In Figure 2, we show the atmospheric neutron spectra for various latitudes obtained with EXPACS for geographical points with an altitude of $10 \mathrm{~km}$ and a longitude of $0^{\circ}$. These data were calculated for the year 2020, period of minimum solar activity.

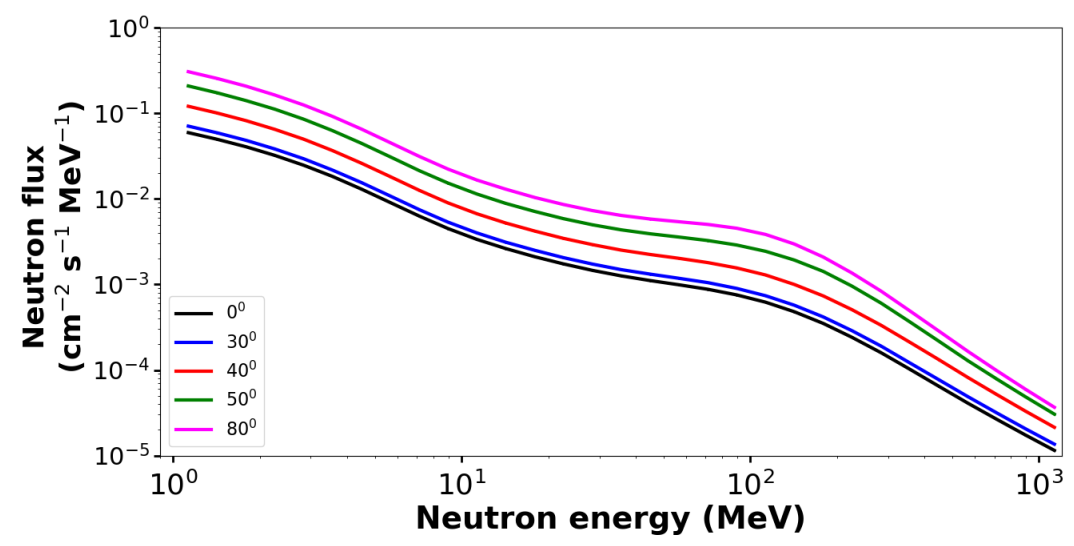

Figure 2. Atmospheric neutron spectra for various latitudes at geographical points with an altitude of $10 \mathrm{~km}$ and a longitude of $0^{\circ}$. See text for more details.

The Geant4 software is a toolkit for the simulation of the passage of particles through matter. It is used for a variety of applications domains, including high-energy physics, astrophysics and space science, medical physics and radiation protection [12]. Geant4 is used extensively in medical physics applications such as particles beam therapy, microdosimetry and radioprotection. The basic extensibility of the toolkit has facilitated its expansion into new user domains, such as biochemistry, material science and non-destructive scanning.

For this simulation we used as source, fluxes of $5 \times 10^{5}$ neutrons with kinetic energies between 1 and $1000 \mathrm{MeV}$. We also used tissues of blood (G4_BLOOD_ICRP, $\left.\rho=1.06 \mathrm{~g} / \mathrm{cm}^{3}\right)$, adipose (G4_ADIPOSE_TISSUE_ICRP, $\rho=0.95 \mathrm{~g} / \mathrm{cm}^{3}$ ), bone (G4_BONE_CORTICAL_ICRP, $\rho=1.92 \mathrm{~g} / \mathrm{cm}^{3}$ ) and brain (G4_BRAIN_ICRP, $\rho=1.04 \mathrm{~g} / \mathrm{cm}^{3}$ ) as samples obtained from Geant 4 material's database, with a spherical geometry with a radius of $5 \mathrm{~cm}$. These samples are tissues equivalent reported by The International Commission on Radiological Protection (ICRP). The ICRP is the primary body in protection against ionising radiation, it is a registered charity and is thus an independent non-governmental organisation created by the 1928 International Congress of Radiology to advance for the public benefit the science of radiological protection [13].

With the help of Geant4 software, we got the RD that neutrons deposit in the different sample tissues as a function of neutron's energy, the neutrons were injected isotropically to each of the samples. We arbitrarily choose geographic points, all of them with the same longitude $\left(0^{\circ}\right)$, at these points, we calculated the RD that neutrons deposited in the samples for different altitudes and different latitudes.

To obtain the RD in the tissues, we used the spectra shown in Figures 1 and 2 to calculate the neutron flux for each energy of interest, in addition, these fluxes were calculated for periods of one hour over the spherical area of the samples, later we multiplied the neutron flux and the RD deposited per neutron, for each energy, finally we added all products to obtain the RD deposited in the tissues.

\section{Results and Discussion}

When calculating the RD that neutrons deposit in the different sample tissues, under the conditions mentioned in the previous section, we obtained the statistical error less than $1 \%$ for each calculated value. Since statistical errors are relatively small, we decided not to point them out in Figure 3 that we present in this section.

In Figure 3, we show the RD that neutrons deposit in the samples as a funtion of their kinetic energy. In this figure, we can see three sections with different behavior of the RD deposited in the samples as a function of the energy of the neutrons; first, for energies of 1 
to $20 \mathrm{MeV}$ the RD curves show an increase with the energy of the neutrons; second, for energies of 20 to $500 \mathrm{MeV}$ the RD curves are approximately flat; finally, for greater energies at $500 \mathrm{MeV}$ the RD curves show an increase with the energy of the neutrons.

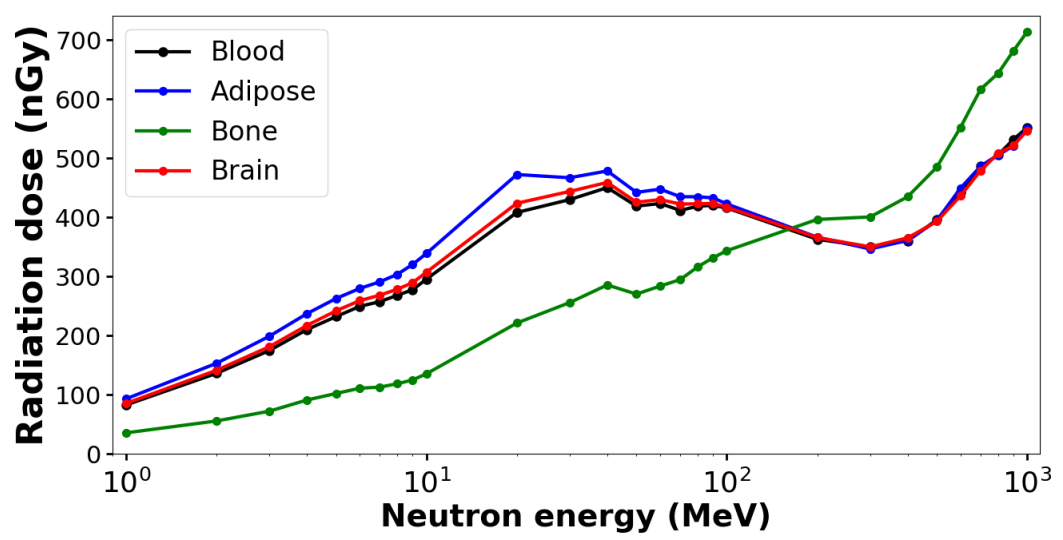

Figure 3. Radiation dose deposited by $5 \times 10^{5}$ neutrons to each energy in the tissue samples. This result was obtained by a numerical simulation.

In Figure 3, we can also observe that, neutrons with energy less than $200 \mathrm{MeV}$ deposited a less RD in the bone sample compared with the RD deposited in the other samples, whereas that, the RD deposited in the adipose sample is greater than the RD deposited in blood and brain samples; this is due to the chemical composition of the tissues, for adipose tissue the dominant element is carbon, for blood and brain tissues is oxygen and for bone tissue are oxygen together with calcium. Neutrons with energy equal or greater than $200 \mathrm{MeV}$ deposited a higher $\mathrm{RD}$ in the bone sample compared with the RD deposited in the other samples, this occurs because the cross section of the neutron varies with the kinetic energy of this particle.

Considering the RD deposited by the neutrons in the bone tissue sample as a reference, in the range of energies from 1 to $100 \mathrm{MeV}$, the RD deposited in the blood, adipose and brain tissue samples are $167.9 \%, 182.8 \%$ and $172.3 \%$ respectively; while, for the range of energies between 200 and $1000 \mathrm{MeV}$, the RD deposited in the blood, adipose and brain tissue samples are $81.0 \%, 80.8 \%$ and $80.5 \%$ respectively.

The neutron fluxes as a function of the altitude and the geographical latitude were calculated under the conditions mentioned in the previous section. In Figure 4, we show the $\mathrm{RD}$ that neutron with energies between 1 and $1000 \mathrm{MeV}$ deposited in the tissue samples for an altitude between 0 and $10 \mathrm{~km}$ above sea level. To avoid overlapping of the curves, we have multiplied them by a factor shown in their respective labels.

In Figure 4, we can see that the RD deposited in the tissue samples is minimal at sea level and increases with height, the reason for this is that the neutron flux increases with increasing height. All curves have the same behavior. From the data obtained from the simulation and considering as reference the RD deposited by neutrons at $0 \mathrm{~km}$ above sea level, for an altitude of $4 \mathrm{~km}$, corresponding approximately to an altitude of high mountain, the $\mathrm{RD}$ is increased $\sim 19$ times, while, for an altitude of $10 \mathrm{~km}$, corresponding to an altitude of commercial flights, the $\mathrm{RD}$ is increased $\sim 156$ times. 


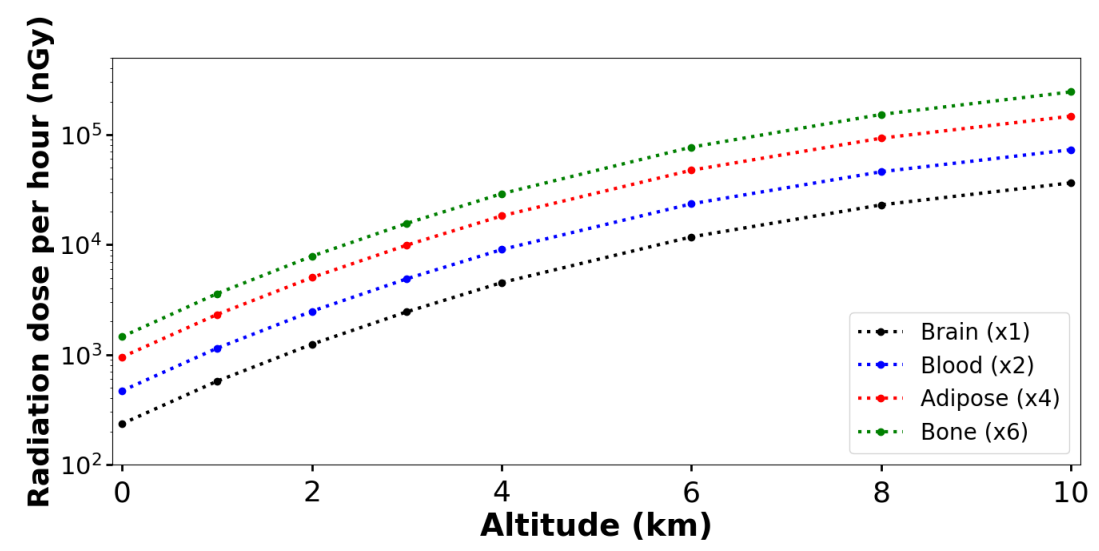

Figure 4. Radiation dose deposited in blood, adipose, brain and bone tissue samples by atmospheric neutrons with energies between 1 and $1000 \mathrm{MeV}$, as a function of the altitude for a latitude of $0^{\circ}$ and a longitude of $0^{\circ}$. This result was obtained by a numerical simulation, the RD was calculated for a period of one hour in spherical samples with a radius of $5 \mathrm{~cm}$. See text for more detail.

In Figure 5, we show the RD that neutron with energies between 1 and $1000 \mathrm{MeV}$ deposited in the tissue samples as a function of the latitude for an altitude of $10 \mathrm{~km}$ above sea level and a longitude of $0^{\circ}$. To avoid overlapping of the curves, we have multiplied them by a factor shown in their respective labels. We can see that the RD deposited in the tissue samples is lower in equatorial zones $\left(\sim 0^{\circ}\right)$; the above is due to the fact that in these zones the rigidity cutoff is maximum, containing lower flux of cosmic rays and consequences a lower production of atmospheric neutrons. In polar zones $\left(\sim 80^{\circ}\right)$, the flux of cosmic rays is greater due to the low-rigidity cutoff which implies a greater production of atmospheric neutrons and consequently a higher RD deposited in the tissue samples. The RD in polar zones is approximately 4.3 times higher than the RD in equatorial zones for an altitud of $10 \mathrm{~km}$.

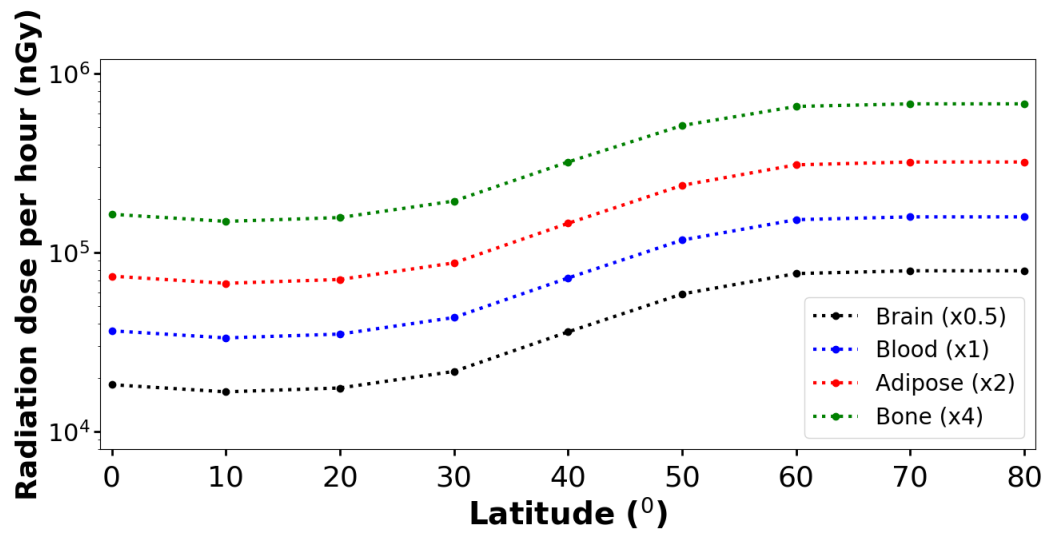

Figure 5. Radiation dose deposited in blood, adipose, brain and bone tissue samples by atmospheric neutrons with energies between 1 and $1000 \mathrm{MeV}$, as a function of the latitude for a altitude of $10 \mathrm{~km}$ and a longitude of $0^{\circ}$. This result was obtained by a numerical simulation, the RD was calculated for a period of one hour in spherical samples with a radius of $5 \mathrm{~cm}$. See text for more detail.

The statistical errors of the data presented in Figures 4 and 5 are dominated by the propagation of the statistical error that is generated when calculating the neutron flux from the energy spectra, therefore, they present variations with the altitude and the latitude. We also found that statistical errors calculated as a percentage are the same for all tissue samples used.

The statistical errors of the RD deposited in each tissue sample of Figure 4 are 5.0\%, $4.9 \%, 4.9 \%, 4.8 \%, 4.9 \%, 5.1 \%, 5.4 \%$ and $5.7 \%$ for altitudes of $0,1,2,3,4,6,8$ and $10 \mathrm{~km}$ above 
sea level, respectively, while for the data in Figure 5 are 5.7\%, 5.7\%, 5.7\%, 5.6\%, 5.4\%, 5.2\%, $5.0 \%, 5.0 \%$ and $5.0 \%$ for latitudes of $0^{\circ}, 10^{\circ}, 20^{\circ}, 30^{\circ}, 40^{\circ}, 50^{\circ}, 60^{\circ}, 70^{\circ}$ and $80^{\circ}$, respectively.

\section{Conclusions}

In this work, and with the help of Geant4 software, first, we calculated the RD that neutrons with energy between 1 and $1000 \mathrm{MeV}$ deposit in blood, adipose, bone and brain tissue samples; second, we calculated the RD that these particles deposit in the tissue samples as a function of different altitudes and latitudes, in both cases, for a longitude of $0^{\circ}$.

When we considered neutrons with a flat spectra $\left(5 \times 10^{5}\right.$ neutrons for each energy) and as a reference the RD deposited by them in the bone tissue sample, we found that in the range of energies from 1 to $100 \mathrm{MeV}$, the RD deposited in the blood, adipose and brain tissue samples is greater by $67.9 \%, 82.8 \%$ and $72.3 \%$ respectively. On the other hand, for the range of energies between 200 and $1000 \mathrm{MeV}$, the RD deposited in the blood, adipose and brain tissue samples is lower by $19.0 \%, 19.2 \%$ and $19.5 \%$ respectively.

When we obtained the atmospheric neutron spectra from [11]; we calculated the RD deposited by these particles in different human tissues as a function of both altitude and latitude and for a longitude of $0^{\circ}$. The results are shown in Figures 4 and 5. In general, we found that the RD deposited by atmospheric neutrons increases with the increase in altitude and latitude, e.g., for an altitude of $4 \mathrm{~km}$ (altitude of high mountain), the RD is increased $\sim 19$ times; while, for an altitude of $10 \mathrm{~km}$ (altitude of commercial flights), the $\mathrm{RD}$ is increased 156 times, respectively; in both cases, regarding the RD at sea level.

Author Contributions: Conceptualization, E.O. and B.M.; Investigation, E.O.; Methodology, E.O.; Software, E.O.; Supervision, B.M. and C.G.; Writing—original draft, E.O.; Writing-review \& editing, B.M., C.G., V.M.M., M.P. and R.G. All authors have read and agreed to the published version of the manuscript.

Funding: This research was funded by Posdoctoral Grant Programa de Becas Posdoctorales de la Universidad Nacional Autónoma de México.

Institutional Review Board Statement: Not applicable, for studies not involving humans or animals. Informed Consent Statement: Not applicable, for studies not involving humans or personal data.

Data Availability Statement: Data are available, contact the corresponding author.

Acknowledgments: The authors thank The European Organization for Nuclear Research (CERN) for Geant4 software.

Conflicts of Interest: The authors declare no conflict of interest. The sponsors had no role in the design, execution, interpretation, or writing of the study.

\section{References}

1. Grieder, P.K.F. Extensive Air Showers. High Energy Phenomena and Astrophysical Aspects. A Tutorial, Reference Manual and Data Book; Springer: Berlin/Heidelberg, Germany, 2010; pp. 6-16.

2. Grieder, P.K.F. Cosmic Rays at Earth: Researcher's Reference Manual Data Book; Elsevier Science B.V.: Amsterdam, The Netherlands, 2001; pp. 77-100.

3. Valdés-Galicia, J.F.; González, L.X. Solar modulation of low energy galactic cosmic rays in the near-earth space environment. Adv. Space Res. 2016, 57, 1294-1306. [CrossRef]

4. Warden, D.; Bayazitoglu, Y. Consideration of backscatter radiation from galactic cosmic rays in spacecraft shielding design. $J$. Thermophys. Heat Transf. 2021, 35. [CrossRef]

5. Gil, A.; Kovaltsov, G.A.; Mikhailov, V.V.; Mishev, A.; Poluianov, S.; Usoskin, I.G. An anisotropic cosmic-ray enhancement event on 07-June-2015: A possible origin. So. Phys. 2018, 293, 154. [CrossRef]

6. Belisheva, N.K.; Lammer, H.; Biernat, H.K.; Vashenuyk, E.V. The effect of cosmic rays on biological systems-An investigation during GLE events. Astrophys. Space Sci. Trans. 2012, 8, 7-17. [CrossRef]

7. Tobiska, W.K.; Atwell, W.; Beck, P.; Benton, E.; Copeland, K.; Dyer, C.; Gersey, B.; Getley, I.; Hands, A.; Holland, M.; et al. Atmospheric radiation measurements and modeling needed to improve air safety. Space Weather 2015, 13, 202-210. [CrossRef]

8. Burgio, E.; Piscitelli, P.; Migliore, L. Ionizing radiation and human health: Reviewing models of exposure and mechanisms of cellular damage. An epigenetic perspective. Int. J. Environ. Res. Public Health 2018, 15, 1971. [CrossRef] [PubMed] 
9. Vulković, B.; Poje, M.; Varga, M.; Radolić, V.; Miklavćić, I.; Faj, D.; Stanić, D.; Planinić, J. Measurements of neutron radiation in aircraft. Appl. Radiat. Isot. 2010, 68, 2398-2402. [CrossRef] [PubMed]

10. Cekanaviciute, E.; Rosi, S.; Costes, S.V. Central nervous system responses to simulated galactic cosmic rays. Int. J. Mol. Sci. 2018, 19, 3669. [CrossRef] [PubMed]

11. EXcel-Based Program for Calculating Atmospheric Cosmic-Ray Spectrum (EXPACS). Available online: https://phits.jaea.go.jp/ expacs/ (accessed on 21 June 2021).

12. Allison, J.; Amako, K.; Apostolakis, J.; Arce, P.; Asai, M.; Aso, T.; Bagli, E.; Bagulya, A.; Banerjee, S.; Barrand, G.; et al. Recent developments in Geant4. Nucl. Instrum. Methods Phys. Res. A 2016, 835, 186-225. [CrossRef]

13. International Commission on Radiological Protection. ICRP Publication 119. Compendium of Dose Coefficients Based on ICRP Publication 60; Ann. ICRP 41; Elsevier: Amsterdam, The Netherlands, 2012. 